\title{
Correction: In thyroxine-replaced hypothyroid postmenopausal women under simultaneous calcium supplementation, switch to oral liquid or softgel capsule L-thyroxine ensures lower serum TSH levels and favorable effects on blood pressure, total cholesterolemia and glycemia
}

\section{Elisabetta Morini ${ }^{1} \cdot$ Antonino Catalano $^{1} \cdot$ Antonino Lasco $^{1} \cdot$ Nunziata Morabito $^{1} \cdot$ Salvatore Benvenga $^{1,2,3}$}

Published online: 28 June 2019

(c) Springer Science+Business Media, LLC, part of Springer Nature 2019

\section{Correction to: Endocrine}

https://doi.org/10.1007/s12020-019-01908-x

Published online 27 March 2019

The original version of this article unfortunately contained mistakes in Fig. 2, Tables 2 and 3.

The corrected figure and tables are given below:

The original article can be found online at https://doi.org/10.1007/ s12020-019-01908-x

\section{Salvatore Benvenga}

s.benvenga@live.it

1 Department of Clinical and Experimental Medicine, University of Messina, Building H, 4th floor, via C. Valeria, Messina 98125, Italy

2 Master Program on Childhood, Adolescent and Women's Endocrine Health, University of Messina, Messina 98125, Italy

3 Interdepartmental Program of Molecular \& Clinical Endocrinology, and Women's Endocrine Health, University Hospital Policlinico G. Martino, via Consolare Valeria, Messina 98125, Italy

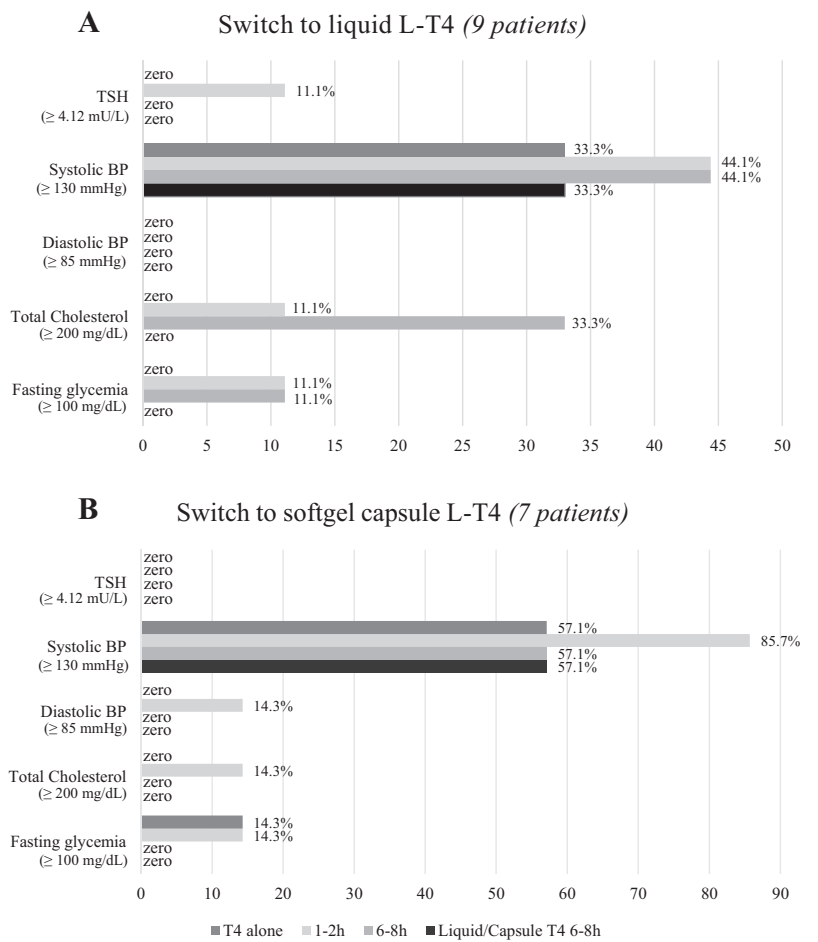

Fig. 2 Percent of patients in categorical TSH levels and TSH-sensitive indices in hypothyroid women, both L-T4 replaced and calcium carbonate supplemented, who were switched from tablet L-T4 to a novel L-T4 formulation (liquid or softgel capsule) 


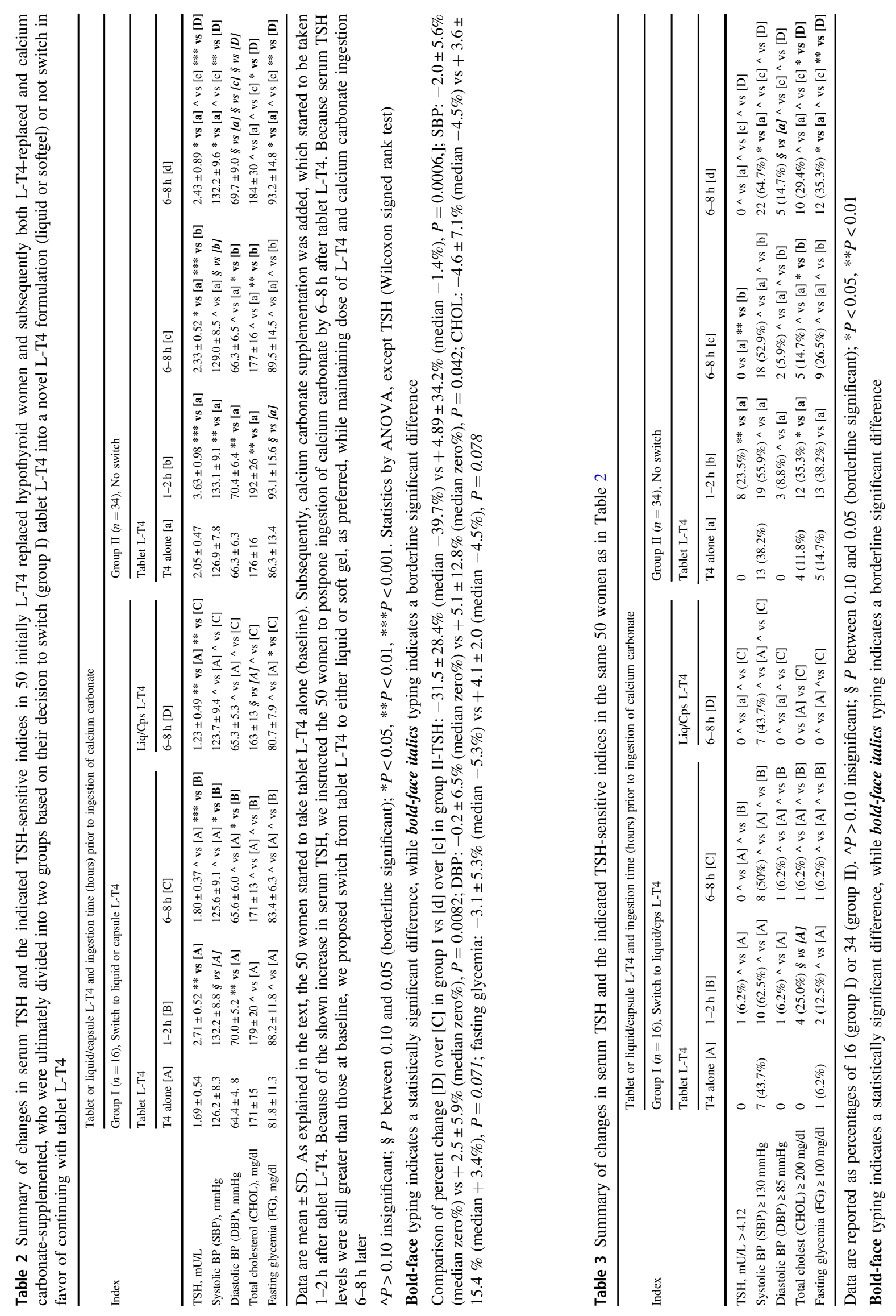

